\title{
Patient-reported outcomes after discontinuation of long-term topical corticosteroid treatment for atopic dermatitis: a targeted cross-sectional survey
}

This article was published in the following Dove Press journal:

Drug, Healthcare and Patient Safety

7 April 2015

Number of times this article has been viewed

\author{
Naoko Takahashi-Ando' \\ Mark A Jones ${ }^{2}$ \\ Shigeki Fujisawa ${ }^{3}$ \\ Rokuro Hama ${ }^{4}$ \\ 'Department of Applied Chemistry, \\ Faculty of Science and Engineering, \\ Toyo University, Saitama, Japan; \\ ${ }^{2}$ School of Population Health, The \\ University of Queensland, Herston, \\ Queensland, Australia; ${ }^{3} \mathrm{Fujisawa}$ \\ Dermatology Clinic, Tokyo, Japan; \\ ${ }^{4} J a p a n$ Institute of Pharmacovigilance, \\ Osaka, Japan
}

Background: Topical corticosteroid (TCS) treatment is widely prescribed for atopic dermatitis (AD). However, TCS treatment is associated with tachyphylaxis, and discontinuation after longterm use may cause exacerbation of symptoms. Some AD patients are reluctant to use TCS.

Objective: To evaluate patient-reported short- and long-term outcomes after discontinuation of TCS treatment for AD.

Methods: Questionnaires were distributed to adult AD patients $(n=1,812)$ of doctors who did not recommend TCS as first-line therapy for patients who preferred to avoid TCS. Data collected included current TCS use, duration of TCS use, past discontinuation of TCS use, exacerbation of symptoms after discontinuation of TCS use, and limitations to daily activities because of AD.

Results: Of 918 respondents, $97.7 \%$ had used TCS, of whom $92.3 \%$ had experienced discontinuation of TCS use. After discontinuation, $63.9 \%$ experienced their most severe AD symptoms ever. The severity of exacerbation of symptoms was significantly correlated with the length of TCS use $(P<0.001)$. Although most respondents who experienced severe exacerbation after TCS discontinuation were not current TCS users, they generally had fewer current limitations to activities than when AD symptoms were at their worst.

Conclusion: Adult Japanese AD patients who experience severe exacerbation of symptoms immediately after discontinuation of TCS use generally improve over time. We suggest caution regarding long-term TCS treatment in AD patients.

Keywords: adverse effects of corticosteroids, symptom exacerbation, rosacea-like dermatitis

\section{Introduction}

The prevalence of atopic dermatitis (AD) has dramatically increased over recent decades. ${ }^{1}$ In Japan, the average reported age of patients with AD has increased, and some patients experience severe and refractory symptoms. ${ }^{2}$ Topical corticosteroid (TCS) treatment is widely prescribed for AD, but may cause skin atrophy, tachyphylaxis, and exacerbation of symptoms after discontinuation of long-term use. ${ }^{3-5}$

The Guidelines for the Management of Atopic Dermatitis in 2000 published by the Japanese Dermatological Association recommend TCS treatment as first-line therapy for AD, combined with secondary treatments such as skin hydration, elimination of aggravating factors, and immunosuppressant medications. ${ }^{6}$ Although the current Japanese treatment guidelines discourage long-term TCS use, especially on the face, ${ }^{7}$ discontinuation may be difficult. Severe exacerbation of symptoms such as rosacea-like dermatitis has been reported after long-term use and discontinuation of TCS in Japanese patients, ${ }^{8-10}$ and in other populations. ${ }^{1-14}$ However, no systematic review is reported for TCS use in $\mathrm{AD}$ patients in the Cochrane Library, and durations
Correspondence: Naoko Takahashi-Ando Department of Applied Chemistry, Faculty of Science and Engineering, Toyo University, 2100 Kujirai,

Kawagoe, Saitama, Japan

$\mathrm{Tel}+8 \mid 49239$ | 384

Fax +8। 4923 23 1031

Email ando_n@toyo.jp 
of randomized placebo-controlled trials for TCS use in AD patients are all short (mostly up to 20 weeks; 44 weeks at the longest ${ }^{15}$ and do not focus on withdrawal outcome (including exacerbation). Under such circumstances, some AD patients have refused to apply TCS, ${ }^{16,17}$ and they tend to be considered as having corticosteroid phobia in medical practice. ${ }^{18}$ Only a few studies have focused on patient-reported outcomes in AD patients who experienced exacerbation of symptoms after discontinuation of TCS use. ${ }^{16,17}$

This study aimed to conduct a large-scale questionnaire survey targeting adult AD patients who had difficulty using TCS or preferred to avoid TCS, to evaluate short- and longterm outcomes after discontinuation of TCS use.

\section{Subjects and methods}

A mail survey was conducted from April 2006 to March 2007, targeting AD patients aged $\geq 16$ years who had difficulty using TCS or preferred to avoid TCS. The questionnaires were distributed by 24 doctors who did not recommend TCS as first-line therapy for patients who preferred to avoid TCS. Questionnaires were distributed to 1,812 patients, who were asked to return the questionnaires by mail.

The questionnaire (see Figure S1) was constructed by Takahashi-Ando, together with medical doctors and AD patients. The following data were collected for each patient: sex, age, past TCS use, duration of TCS use, current TCS use, past and current tacrolimus ointment use, past discontinuation of TCS use, exacerbation of symptoms after discontinuation of TCS use, limitations to daily activities because of AD at the time of the worst symptoms and at the time of the survey, what improved the symptoms of AD if they were under control, and difficult experiences with medical treatment and consulting medical experts (see Figure S1).

Stata/SE (v13.0 for Windows; StataCorp LP, College Station, TX, USA) was used for statistical analysis. Binary logistic regression was used to estimate odds ratios (OR) and $95 \%$ confidence intervals (CI) for binary response variables measured in the survey. Ordinal logistic regression was used to estimate OR and 95\% CI for ordinal response variables. Wald tests were used to estimate $P$-values to test the null hypothesis of no association between response and explanatory variables. Contrasts were used to compare the effects of the five categories of duration of TCS use on severity of exacerbation. The contrasts were based on comparing each category with the observationweighted mean of subsequent categories. The proportional odds assumption for ordinal regression was tested using a likelihood ratio test.

\section{Results}

Completed questionnaires were received from 918 respondents (393 males, 523 females, two unknown). The age of distribution of respondents was as follows: 16-20 years, $8.4 \%$; $21-30$ years, $41.5 \% ; 31-40$ years, $38.8 \%$; $41-50$ years, $8.4 \%$; $\geq 51$ years, $2.4 \%$; and unknown, $0.5 \%$.

TCS use at any time was reported by $97.7 \%$ of the respondents (897/918). Duration of TCS use was as follows: $<1$ year, $7.7 \%$; 1-5 years, $23.5 \%$; 5-10 years, 22.7\%; 10-20 years, $25.8 \%$; $>20$ years, $14.6 \%$; unknown, 5.7\%. Among respondents who reported TCS use, 92.3\% (828/897) had experienced discontinuation of TCS use, 83.2\% (746/897) were not current users of TCS, 53.4\% (479/897) had ever used tacrolimus ointment, and 10.1\% (91/897) were current users of tacrolimus ointment.

Among respondents who had experienced discontinuation of TCS use, 63.9\% (529/828) reported experiencing their most severe AD symptoms ever immediately after discontinuation (Table 1). Figure 1 shows that a longer duration of TCS use was associated with greater exacerbation of symptoms after discontinuation. Statistical analysis showed a $27 \%$ increased odds of reporting more severe exacerbation of symptoms by increasing duration of TCS use (OR: 1.27, 95\% CI: $1.12-1.44, P<0.001)$. There was no evidence of nonproportionality of odds across the categories of the response $(P=0.28)$. We also investigated whether the effect of duration of TCS use on severity of exacerbation after discontinuation

Table I Current use of TCS according to the severity of exacerbation of symptoms after discontinuation of TCS use $n=828$

\begin{tabular}{lllll}
\hline $\begin{array}{l}\text { Severity of exacerbation after } \\
\text { discontinuation of TCS use }\end{array}$ & Total & Current TCS use & & \\
\cline { 3 - 5 } & & Yes: n (\%) & No: n (\%) & Unknown: n (\%) \\
\hline A. Most severe symptoms ever & 529 & $42(7.9 \%)$ & $484(91.5 \%)$ & $3(0.6 \%)$ \\
B. Substantial exacerbation & 153 & $25(16.3 \%)$ & $125(81.7 \%)$ & $3(2.0 \%)$ \\
C. Moderate exacerbation & 88 & $23(26.1 \%)$ & $63(71.6 \%)$ & $2(2.3 \%)$ \\
D. No change in symptoms & 45 & $15(33.3 \%)$ & $30(66.7 \%)$ & $0(0 \%)$ \\
E. Other/unknown & $10 / 3$ & $3(23.1 \%)$ & $8(61.5 \%)$ & $2(15.4 \%)$ \\
\hline
\end{tabular}

Note: a Odds of reporting current TCS use decreased by $47 \%$ for each increase in severity category (OR: $0.53,95 \% \mathrm{Cl}: 0.44-0.65, P<0.001$; excluding other/unknown). Abbreviations: $\mathrm{Cl}$, confidence interval; OR, odds ratio; TCS, topical corticosteroids. 


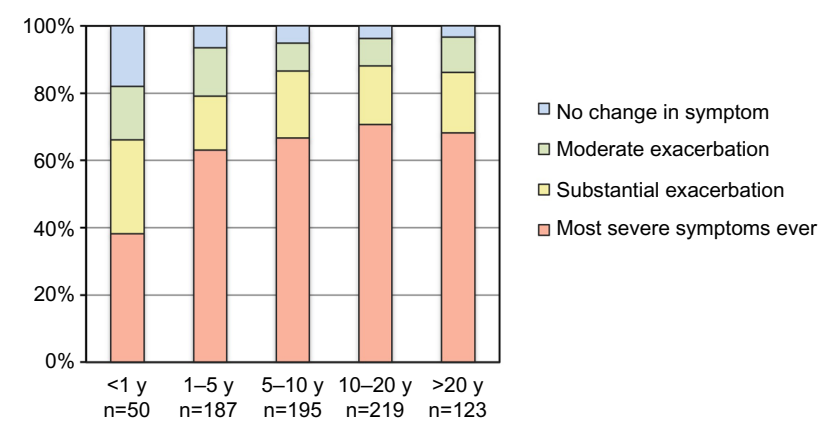

Figure I Duration of use of TCS and severity of worsening at withdrawal of TCS. Note: Subjects with unknown duration of TCS use or unknown severity of exacerbation were excluded from this analysis.

Abbreviations: TCS, topical corticosteroids; $y$, years.

is confounded by age in a multivariable ordinal logistic regression. Results showed that the duration of TCS use is not confounded by age (OR changed minimally from 1.27 to $1.24)$ and that age is not an independent predictor of severity of exacerbation $(P=0.12)$.

In an additional analysis, we investigated further the relationship between duration of TCS use and severity of exacerbation after discontinuation by performing contrasts between the five categories of duration. Results showed that $<1$ year duration is statistically different from durations of $>1$ year $(P<0.0001)$; $1-5$ years duration may be different from durations $>5$ years $(P=0.058)$; however, there was no evidence of a difference between durations longer than 5 years $(P>0.4)$.

Patients who experienced more severe exacerbation after discontinuation of TCS use were significantly less likely to be current users of TCS and tacrolimus ointment than those who experienced less severe exacerbation (Tables 1 and S1). Statistical analysis showed that increasing severity of exacerbation of symptoms immediately after discontinuation of TCS use was associated with a $47 \%$ decreased odds of current use of TCS (OR: $0.53,95 \%$ CI: $0.44-0.65, P<0.001)$ and a $23 \%$ decreased odds of current use of tacrolimus (OR: 0.77, 95\% CI: 0.60-0.98, $P=0.035$; Table S1).

Among respondents who reported their most severe AD symptoms ever immediately after discontinuation of TCS use, $86.2 \%$ (456/529) were not current users of either TCS or tacrolimus ointment. In these subjects, limitations to daily activities because of AD were evaluated (Table 2). At the time of their worst symptoms (immediately after discontinuation of TCS use), 37.3\% reported difficulty with indoor movement and only 5.0\% reported no limitations to any of the activities surveyed. At the time of the survey, $48.5 \%$ reported no limitations to any of the activities.
Table 2 Limitations to daily activities because of $A D$ among $A D$ patients who reported their most severe $A D$ symptoms after TCS discontinuation and no current use of TCS nor tacrolimus ointment $n=456$

\begin{tabular}{lll}
\hline & $\begin{array}{l}\text { At the time of } \\
\text { worst symptoms }\end{array}$ & Current \\
\hline Indoor movement & $170(37.3 \%)$ & $16(3.5 \%)$ \\
Leaving the home & $364(79.8 \%)$ & $134(29.4 \%)$ \\
Use of public transportation & $305(66.9 \%)$ & $91(20.0 \%)$ \\
Commuting $^{\mathrm{a}}$ & $325(71.3 \%)$ & $180(39.5 \%)$ \\
None of the above & $23(5.0 \%)$ & $221(48.5 \%)$ \\
\hline
\end{tabular}

Notes: aSubjects who did not commute were asked to imagine what it would be like to commute. Multiple answers were allowed.

Abbreviations: $A D$, atopic dermatitis; TCS, topical corticosteroids.

Among those whose symptoms were under control $(n=466)$, the following answers were obtained for the reasons of improvement (multiple choice): spontaneous recovery $(n=64)$, change of environment $(n=57)$, change of diet ( $n=180)$, exercise ( $n=61$ ), use of TCS or tacrolimus ointment $(n=19)$, avoidance of TCS use $(n=310)$, herbal treatments or other alternative remedies $(n=151)$, and other $(\mathrm{n}=107)$. There was an association between 'avoidance of TCS use' and AD symptoms immediately after discontinuation, with $79.0 \%(226 / 286)$ of those who experienced their worst symptoms ever after discontinuation, 68.4\% (54/79) of those who experienced substantial exacerbation, $51.2 \%$ (21/41) of those who experienced moderate exacerbation, and $15.0 \%(3 / 20)$ of those who experienced no change, considering 'avoidance of TCS use' to be a reason for improvement of their symptoms. Statistical analysis showed that increasing severity of exacerbation of symptoms immediately after discontinuation of TCS use is associated with a more than two-fold increased odds of reporting 'avoidance of TCS' as one of the reasons for improvement of symptoms (OR: 2.2, 95\% CI: 1.7-2.8, $P<0.001)$.

Many patients listed difficulties they had experienced in consulting medical experts and using medical treatments, including: long waiting time prior to consultation, short consultation time, no explanation of how to use medications, medical experts unwilling to listen to patients' experiences, and unwanted treatments being recommended.

\section{Discussion}

This is the first large-scale study focusing on self-reported short- and long-term outcomes in adult AD patients after discontinuation of TCS use. The severity of exacerbation of symptoms was significantly correlated with the length of TCS use. Our findings are consistent with those of a previous study that reported a correlation between duration of TCS 
use and exacerbation of facial AD after discontinuation. ${ }^{19}$ Interestingly, the effect of discontinuing TCS use on severity of exacerbation increased with increasing durations of use over the first 5 years but then appeared to plateau, suggesting at least a partial explanation as to why some patients experienced TCS-induced withdrawal exacerbation, while others did not.

In Japan, it is believed that major reasons for stopping TCS are exaggerated information of adverse effects made by the media and sellers of unproven alternatives to TCS, ${ }^{20}$ and they were simply taken as corticosteroid phobia. However, this study found that those who experienced more severe aggravation after discontinuation were significantly less likely to be using TCS at the time of the survey $(P<0.001)$, indicating that reluctance to use TCS may be associated with the patient's own experience of TCS use. Thus, when a patient is reluctant to use TCS, physicians should consider the possibility that he/she has experienced TCS-induced adverse effects.

According to comments in the free description space on the questionnaire, many patients experienced lower effectiveness of TCS after continuous use, which induced them to discontinue TCS. Symptoms such as intense pruritus, redness, burning, and edema after discontinuation of TCS use were severe enough to induce suicidal thoughts in some subjects. Respondents reported symptoms consistent with the previously reported 'red skin syndromes'"11 or rosacea-like dermatitis. ${ }^{13,14}$ Many patients experienced severe exacerbation not only on their face but also other areas of their body, and in some cases, symptoms spread to areas where patients had never applied TCS, suggesting that their symptoms were not simple rosacea-like dermatitis, which typically localizes on the face. Dermatitis occurring on areas where patients never applied TCS could possibly be attributed to corticosteroids being absorbed and systemically distributed. Such cases of dermatitis have also been reported by patients using inhaled corticosteroids $^{21}$ or orally administered corticosteroids. ${ }^{22}$

Many of these patients recovered eventually, and believed that continuing to avoid TCS use was necessary for recovery. Many also expressed frustration that conventional doctors pressured them to continue TCS use. Such patients may stop consulting conventional doctors, and may therefore be underrepresented in some clinical studies. The majority of these patients were not using tacrolimus ointment to control their symptoms.

A limitation of this study is potential selection bias. The response rate was $51 \%$, which could induce selection bias, although the rate is typical for a mail survey. As this study focused on adult AD patients who had difficulty using TCS or preferred to avoid TCS, the population surveyed was not a representative sample of AD patients in Japan and they may have been more liable to experience TCS-induced withdrawal exacerbation than general AD patients. However, data from a multicenter retrospective analysis of 1,271 general AD patients (210 infants, 546 children, and 515 adolescents and adults) by Furue et al, ${ }^{23}$ showed that after 6 months of TCS treatment, $3 \%$ had worse symptoms and 58\% showed no improvement. In the subgroup of adult cases, the proportion of those with worse symptoms and no improvement was higher than those in the subgroups of children and infants. These results suggest that TCS treatment may not be beneficial for some general AD patients, especially adult cases. Furthermore, it may lead to worsening symptoms and long-term use could potentially result in TCS-induced adverse effects.

In conclusion, the findings of this study show that a substantial number of adult AD patients experience exacerbation of symptoms after discontinuation of TCS use, especially after long-term use, and many patients who experience such an exacerbation subsequently improve. Physicians are urged to be cautious of recommending long-term TCS use, and to be aware of the possibilities of TCS-induced adverse effects when a patient has become reluctant to use TCS. Since TCS is the first-line therapy worldwide, the adverse effects shown in our study could occur in general AD patients; thus, studies that investigate the outcome of TCS withdrawal in general AD patients are urgently needed.

\section{Acknowledgments}

We greatly appreciate the cooperation of the dermatologists, physicians, AD patients, and supporting help groups. This study was supported by the Takagi Fund of Citizen Science. Writing assistance was provided by Edanz Group Global Ltd (Wanchai, Hong Kong).

\section{Disclosure}

The authors report no conflicts of interest in this work.

\section{References}

1. Williams H, Robertson C, Stewart A, et al. Worldwide variations in the prevalence of symptoms of atopic eczema in the International Study of Asthma and Allergies in Childhood. J Allergy Clin Immunol. 1999; 103(1 Pt 1):125-138.

2. Nishioka K. Atopic eczema of adult type in Japan. Australas J Dermatol. 1996;37 Suppl 1:S7-S9.

3. Kligman AM, Frosch PJ. Steroid addiction. Int J Dermatol. 1979;18(1): 23-31.

4. Zheng PS, Lavker RM, Lehmann P, Kligman AM. Morphologic investigations on the rebound phenomenon after corticosteroid-induced atrophy in human skin. J Invest Dermatol. 1984;82(4):345-352. 
5. Hengge UR, Ruzicka T, Schwartz RA, Cork MJ. Adverse effects of topical glucocorticosteroids. J Am Acad Dermatol. 2006;54(1):1-15; quiz 16-18.

6. Kawashima M, Takigawa M, Nakagawa H, et al. Guidelines for therapy for atopic dermatitis. Jpn J Dermatoallergol. 2000;110(7):1099-1104. [Japanese with English abstract].

7. Saeki H, Furue M, Furukawa F, et al. Guidelines for management of atopic dermatitis. J Dermatol. 2009;36(10):563-577.

8. Fukaya M. Improvement of atopic dermatitis after discontinuation of topical corticosteroid treatment. Arch Dermatol. 2000;136(5):679-680.

9. Tamaki A, Ohashi A, Ishida T, Nakamura M. Treatment without steroid ointment for adult-type atopic dermatitis. Jpn J Dermatoallergol. 1993;1:230-234. [Japanese with English abstract].

10. Fukaya M, Sato K, Sato M, et al. Topical steroid addiction in atopic dermatitis. Drug Healthc Patient Saf. 2014;6:131-138.

11. Rapaport M, Rapaport V. The red skin syndromes: corticosteroid addiction and withdrawal. Expert Rev Dermatol. 2006;1(4):547-561.

12. Chen AY, Zirwas MJ. Steroid-induced rosacealike dermatitis: case report and review of the literature. Cutis. 2009;83(4):198-204.

13. Rathi SK, Kumrah L. Topical corticosteroid-induced rosacea-like dermatitis: a clinical study of 110 cases. Indian J Dermatol Venereol Leprol. 2011;77(1):42-46.

14. Bhat YJ, Manzoor S, Qayoom S. Steroid-induced rosacea: a clinical study of 200 patients. Indian J Dermatol. 2011;56(1):30-32.
15. Hanifin J, Gupta AK, Rajagopalan R. Intermittent dosing of fluticasone propionate cream for reducing the risk of relapse in atopic dermatitis patients. Br J Dermatol. 2002;147(3):528-537.

16. Fukaya $M$. Why do patients with atopic dermatitis refuse to apply topical corticosteroids? Dermatology. 2000;201(3):242-245.

17. Ando N. Testimony of One Thousand Patients of Atopic Dermatitis. Tokyo, Japan: Kodomono Miraisya; 2008. [Japanese].

18. Aubert-Wastiaux H, Moret L, Le Rhun A, et al. Topical corticosteroid phobia in atopic dermatitis: a study of its nature, origins and frequency. Br J Dermatol. 2011;165(4):808-814.

19. Katayama I, Yokoyama A, Matsunaga T, Yokozeki H, Nishioka K. Non-steroid therapy for refractory facial dermatitis in adult atopic dermatitis. Jpn J Dermatol. 1994;104:875-880. [Japanese with English abstract].

20. Takehara K. Atopy Business. Tokyo, Japan: Bungeishunju Ltd; 2000. [Japanese].

21. Tashkin DP, Murray HE, Skeans M, Murray RP. Skin manifestations of inhaled corticosteroids in COPD patients: results from Lung Health Study II. Chest. 2004;126(4):1123-1133.

22. Tomita Y, Tagami H. Steroid-withdrawal rosacea-like dermatitis. J Dermatol. 1989;16(4):335-337.

23. Furue M, Terao H, Rikihisa W, et al. Clinical dose and adverse effects of topical steroids in daily management of atopic dermatitis. $\mathrm{Br} J$ Dermatol. 2003;148(1):128-133. 


\section{Supplementary materials}

Table SI Current use of tacrolimus according to the severity of exacerbation of symptoms after discontinuation of TCS use $\mathrm{n}=828$.

\begin{tabular}{lllll}
\hline $\begin{array}{l}\text { Severity of exacerbation after } \\
\text { discontinuation of TCS use }\end{array}$ & Total & \multicolumn{3}{l}{ Current tacrolimus use $^{\mathbf{a}}$} \\
\cline { 3 - 5 } & & Yes: $\mathbf{n}(\%)$ & No: $\mathbf{n}(\%)$ & Unknown: $\mathbf{n}(\%)$ \\
\hline A. Most severe symptoms ever & 529 & $35(6.6 \%)$ & $486(91.9 \%)$ & $8(1.5 \%)$ \\
B. Substantial exacerbation & 153 & $15(9.8 \%)$ & $133(86.9 \%)$ & $5(3.3 \%)$ \\
C. Moderate exacerbation & 88 & $13(14.8 \%)$ & $73(83.0 \%)$ & $2(2.3 \%)$ \\
D. No change in symptoms & 45 & $4(8.9 \%)$ & $41(91.9 \%)$ & $0(0 \%)$ \\
E. Other/unknown & $10 / 3$ & $5(38.5 \%)$ & $8(61.5 \%)$ & $0(0 \%)$ \\
\hline
\end{tabular}

Note: ${ }^{a}$ Odds of reporting current tacrolimus use decreased by $23 \%$ for each increase in severity category $(\mathrm{OR}$ : $0.77,95 \% \mathrm{Cl}: 0.60-0.98, P=0.035$; excluding other/ unknown).

Abbreviations: $\mathrm{Cl}$, confidence interval; OR, odds ratio; TCS, topical corticosteroids.

A) Sex: Male; Female

B) Age: 16-20 years; $21-25$ years; $26-30$ years; $31-35$ years; $36-40$ years; $41-45$ years; $46-50$ years; $51-60$ years; 61 years or older

C) Have you ever used topical corticosteroids? Yes; No; Don't know

D) How long have you used topical corticosteroids? Less than 1 year; 1-5 years; 5-10 years; 10-20 years; longer than 20 years; Don't know

E) Are you currently using topical corticosteroids? Yes; No

F) Have you ever used tacrolimus ointment? Yes; No

G) Are you currently using tacrolimus ointment? Yes; No

H) Have you ever attempted to stop using topical corticosteroids? Yes; No

I) What happened when you stopped using topical corticosteroids? Most severe symptoms in my life; Substantial exacerbation of symptoms, but not the most severe symptoms in my life; Moderate exacerbation of symptoms/within usual exacerbation; No change in symptoms; Other/Unknown

J) Please indicate which daily activities were limited by your atopic dermatitis when the symptoms were at their worst. Indoor movement; Leaving the home; Use of public transportation; Commuting (if you did not commute, please imagine what it would have been like to do so); None of the above

K) Please indicate which daily activities are currently limited by your atopic dermatitis. Indoor movement; Leaving the home; Use of public transportation; Commuting (if you do not commute, please imagine what it would be like to do so); None of the above

L) If you feel that you can control your symptoms of atopic dermatitis, what improved your symptoms? Multiple answers are allowed. Spontaneous recovery; Change of environment; Change of diet; Exercise; Use of topical corticosteroids or tacrolimus ointment; Avoidance of topical corticosteroids; Herbal treatments or other alternative remedies; Other

M) Please explain any difficulties you have experienced with the medical treatment of your atopic dermatitis and consulting medical experts (in free description space)

Figure SI Questionnaire.

Drug, Healthcare and Patient Safety

Dovepress

\section{Publish your work in this journal}

Drug, Healthcare and Patient Safety is an international, peer-reviewed open-access journal exploring patient safety issues in the healthcare continuum from diagnostic and screening interventions through to treatment, drug therapy and surgery. The journal is characterized by the rapid reporting of reviews, original research, clinical, epidemiological and post-marketing surveillance studies, risk management, health literacy and educational programs across all areas of healthcare delivery. The manuscript management system is completely online and includes a very quick and fair peer-review system. Visit http://www.dovepress.com/ testimonials.php to read real quotes from published authors. 